\title{
On the phase diagram of 2d Lorentzian Quantum Gravity
}

\author{
Jan Ambjørn ${ }^{a}$, K. N. Anagnostopoulos ${ }^{a}$ and R. Loll ${ }^{b}$ \\ a Niels Bohr Institute, Blegdansvej 17, DK-2100 Copenhagen $\emptyset$, Denmark \\ bAlbert-Einstein-Institut, Am Mühlenberg 5, D-14476 Golm, Germany
}

The phase diagram of $2 \mathrm{~d}$ Lorentzian quantum gravity (LQG) coupled to conformal matter is studied. A phase transition is observed at $c=c_{\text {crit }}\left(1 / 2<c_{\text {crit }}<4\right)$ which can be thought of as the analogue of the $c=1$ barrier of Euclidean quantum gravity (EQG). The non-trivial properties of the quantum geometry are discussed.

\section{INTRODUCTION}

Recently a new model of $2 \mathrm{~d}$ quantum gravity has been proposed [1]. It is defined using dynamical triangulations from a subclass of diagrams which can be given a causal structure. Such diagrams are generated by gluing together one dimensional time-slices or "universes" (in our case a set of vertices connected by space-like links forming a diagram with the topology of a circle) with time-like links such that they form a triangulated surface. Vertices connected by timelike links are causally related and a unique time can be assigned to the vertices of each time-slice. Such graphs can be given a Lorentzian metric by defining time-like links to have equal negative length squared and space-like to have positive. All triangles have equal area and the volume of spacetime is proportional to the number of triangles $N_{T}$. The system has been found to have a non-trivial continuum limit only at an imaginary value of the cosmological constant $\lambda$. The geometry of space is maximally fluctuating but the system is much smoother than Liouville gravity: By defining the two point function to be

$G(\lambda, t)=\sum_{T \in \mathcal{T}} \mathrm{e}^{-\lambda N_{T}}, \quad \lambda \in \mathbb{R}$,

where the summation is over triangulations $T$ of cylindrical topology with $t$ time-slices, one finds that the Hausdorff dimension $d_{H}$ of the system is 2. This is entirely due to the imposition of the causal structure: If one allows the creation of baby universes the system becomes the ordi- nary EQG model. If conformal matter of central charge $c$ is coupled to the system, it has been found in the case of one Ising model [2] that the coupling is weak for $c \leq 1 / 2$ and the bulk properties of geometry do not change (e.g. $d_{H}=2$ ). The critical matter system belongs to the Onsager universality class. When 8 Ising spins are coupled to gravity, a qualitatively different behaviour emerges. The system undergoes a phase transition for some $1 / 2<c_{\text {crit }}<4$ [3] to a phase where we observe anomalous scaling between the typical length scale $L$ and typical time scale $T$. It is found that $\operatorname{dim}[L]=2 \operatorname{dim}[T]$ and that $d_{H}=3$. The latter is a "cosmological" Hausdorff dimension. The former is a relation which has been found to hold for the non-singular part of the quantum geometry of EQG for any value of $c \leq 1$. The short distance behaviour of space-time is given by a different fractal dimension $d_{h}=2$. The quantum geometry is different at different scales and has a more complex structure. The matter critical exponents are Onsager. We conclude that the matter coupling to geometry in our model is much weaker than in the case of EQG so that the large $c$ phase has non-degenerate, interesting continuum limit for the quantum geometry. Nothing dramatic happens from the point of view of matter but the geometry undergoes a qualitative change and even shares some features with the non-singular part of EQG in the $c<1$ phase. This is the first time where one has a model which demonstrates explicitly that the strong coupling of matter and geometry in Liouville gravity is 


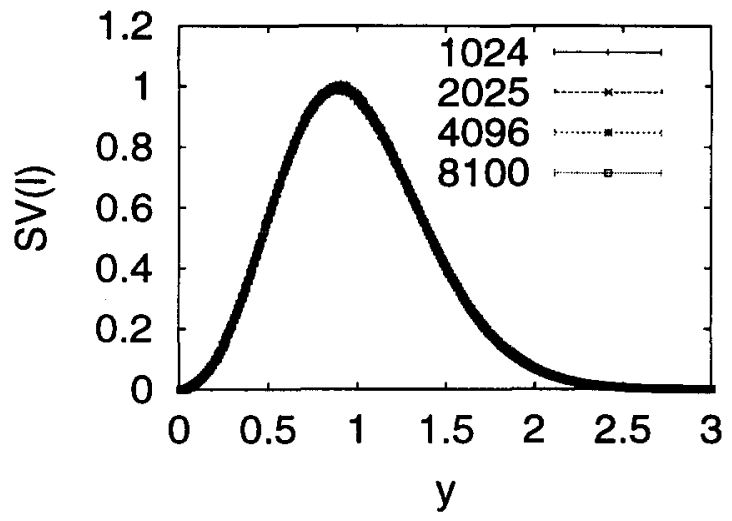

Figure 1. The $S V(l)$ distributions for the $c=1 / 2$, $\tau=1$ system. $y=l / N^{1 / \delta_{h}}$

entirely due to the (a priori) presence of babyuniverses.

\section{NUMERICAL RESULTS}

We simulate triangulated surfaces with the topology of the torus with $N_{T}$ triangles and $N=$ $N_{T} / 2$ vertices [2,3]. The temporal length (number of time slices) is $t$ and we choose $N=t^{2} / \tau$ for $\tau=1,2,3$ and 4 . The geometry is updated with the move described in [2] and the Ising spins with the Swendsen-Wang algorithm. The partition function for $n$ Ising models is

$G(\lambda, t, \beta)=\sum_{T \in \mathcal{T}} \mathrm{e}^{-\lambda N_{T}} Z_{T}^{n}(\beta)$.

$Z_{T}(\beta)$ is the partition function of an Ising model with spins placed at the vertices of the graph $T$ at inverse temperature $\beta . n=1,8$ corresponding to $c=1 / 2,4$. We obtain the fixed volume partition function by adding a gaussian volume-fixing term and measuring only on configurations of given volume $N_{T}$. The critical values of $(\lambda, \beta)$ are determined. For $c=0$ they are $\lambda_{c}=\ln 2$, for $c=1 / 2$ they are $\left(\lambda_{c}, \beta_{c}\right)=(0.742(5), 0.2521(1))$ and for $c=4$ they are $\left(\lambda_{c}, \beta_{c}\right)=(1.081(5), 0.2480(4))$ $(\lambda \rightarrow \ln 2$ when $\beta \rightarrow \infty)$. Those values are insensitive to $\tau$. Finite size scaling (FSS) is applied at

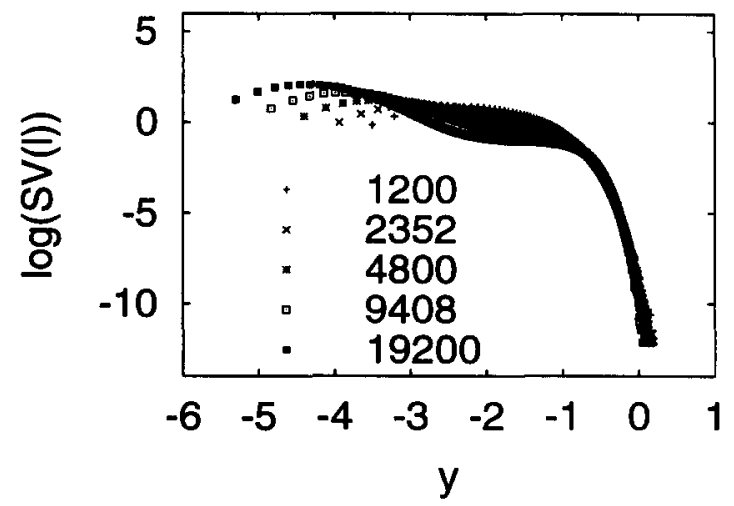

Figure 2. The $S V(l)$ distributions for the $c=4$, $\tau=3$ system. $y=l / N^{1 / \delta_{h}}$

$\left(\lambda_{c}, \beta_{c}\right)$ in order to measure the system's scaling properties.

The first quantity that we measure is the distribution $S V(l)$ of spatial volumes $l$. We expect a scaling behaviour

$S V_{N}(l)=F_{S}\left(l / N_{T}^{1 / \delta_{h}}\right)$,

for some function $F_{S} . \delta_{h}$ is related to the fractal dimension $t \sim N_{T}^{1 / d_{H}}$ by $d_{H}=\delta_{h} /\left(\delta_{h}-1\right)$. Here, $t$ is the dynamically generated time extend of the scaling part of space-time. For the $c=1 / 2$ model we find $\delta_{h}=d_{H}=2$ as shown in Fig. 1 . For $c=4$ the scaling behaviour of $S V(l)$ is qualitatively different. As can be seen in Fig. 3, the configurations show a tendency to form a long and thin neck with spatial volume of the order of the cutoff and an extended region that scales according to (2). The volume of the extended part is asymptotically proportional to $N_{T}$. The effect is seen for large enough volumes and finite size effects are minimised by taking $\tau \geq 3$. For $\tau=3$ we find $\delta_{h}=1.54(3)$ (see Fig. 2) and for $\tau=4 \delta_{h}=1.50(3)$. Let us assume for clarity that $\delta_{h}=3 / 2$. Then $d_{H}=3$ and $l \sim t^{2}$ where $l$ is the typical length scale of universes in the extended region and $t$ the typical time scale of their existence. 


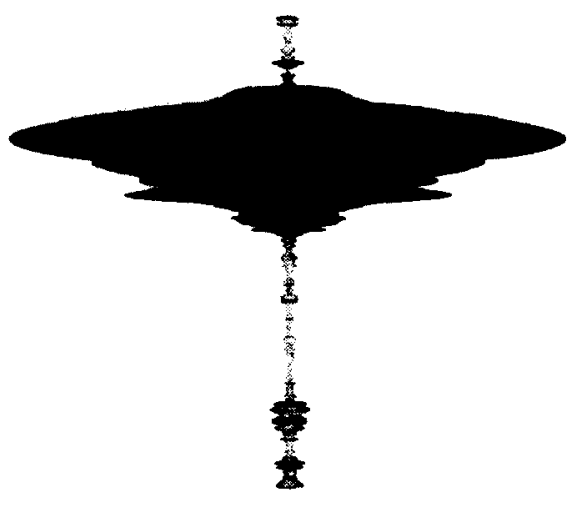

Figure 3. A typical configuration of the $c=1 / 2$, $\tau=3$ system. $N_{T}=73926$.

The above results are supported by measuring the volumes $n_{N}(r)$ of geodesic spherical shells at distance $r$. We expect that $n_{N}(r)=$ $N_{T}^{1-1 / d_{H}} F_{1}(x), x=r / N_{T}^{1 / d_{H}}$ which is known to hold in EQG for all c. $d_{H}$ is the cosmological Hausdorff dimension describing how volume and time have to scale in order to obtain a non trivial continuum limit of the two loop function. On the other hand the short distance behaviour is given by $d_{h}$, where $n_{N}(r) \sim r^{d_{h}-1}$ at scales $r \ll N^{1 / d_{H}}$. For $c=0$ we find analytically that $d_{H}=d_{h}=2$ and for $c=1 / 2$ numerically that $d_{H}=d_{h}=2.00(5)$. For $c=4, n_{N}(r)$ has different scaling behaviour at different length scales. For $r \ll N^{1 / 3}$ we find that $d_{h}=2.1(2)$ as can be seen of Fig. 4. For $r \gg N^{1 / 3}$ the value of the tail of $n_{N}(r)$ is almost independent of $N_{T}$ and

Table 1

The critical exponents for the $c=1 / 2, \tau=1$ and the $c=4, \tau=3$ systems.

\begin{tabular}{cccc}
\hline & & $c=1 / 2$ & $c=4$ \\
\hline$\chi$ & $\frac{\gamma}{\nu d_{H}}$ & $0.89(1)$ & $0.85(1)$ \\
$D_{\ln |m|}$ & $\frac{1}{\nu d_{H}}$ & $0.526(5)$ & $0.520(5)$ \\
$D_{\ln m^{2}}$ & $\frac{1}{\nu d_{H}}$ & $0.525(5)$ & $0.512(5)$ \\
\hline
\end{tabular}

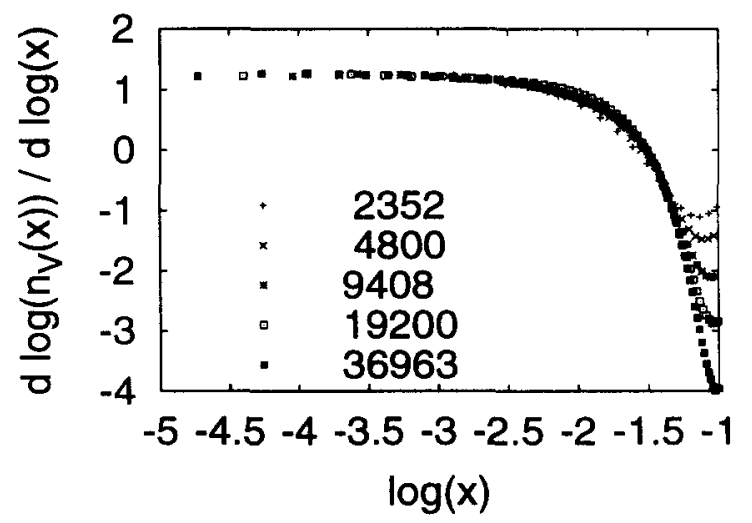

Figure 4. Short distance scaling of $n_{N}(r)$ for $c=$ $4, \tau=3$.

$r$ showing dominance of $1 \mathrm{~d}$ configurations. For $r \sim N^{1 / 3}$ the scaling of the peaks of $n_{N}(r)$ gives $d_{H}=3.07(9)$.

The matter scaling exponents are computed from the scaling behaviour of the magnetic susceptibility $\chi \sim N^{\gamma / \nu d_{H}}$ and $D_{\ln |m|} \equiv \frac{d \ln |m|}{d \beta} \sim$ $N^{1 / \nu d_{H}}$ and $D_{\ln m^{2}} \equiv \frac{d \ln m^{2}}{d \beta} \sim N^{1 / \nu d_{H}}$. The $c=1 / 2$ system clearly belongs to the Ising universality class. For $c=4$ special care has to be taken in order to isolate the critical behaviour of the spin system in the extended region of spacetime [3]. The critical exponents are consistent with Onsager values. Our results are summarized in Table 1.

\section{REFERENCES}

1. J. Ambjørn and R. Loll, Nucl. Phys. B 536 (1998) 407-434, [hep-th/9805108].

2. J. Ambjørn, K.N. Anagnostopoulos and R. Loll, Phys. Rev. D, to appear, [hep-th/9904012].

3. J. Ambjørn, K.N. Anagnostopoulos and R. Loll: Crossing the $c=1$ barrier in $2 d$ Lorentzian Quantum Gravity, to appear. See also http://www.nbi.dk/ ambjorn/lqg2 . 